\title{
Political Violence and Children in the City of Bulawayo
}

\begin{abstract}
The purpose of this research is to explore how political violence threatens the health of young children in the city of Bulawayo, Zimbabwe. Using a difference-in-differences regression methodology, we reveal the damaging consequences of political violence on Zimbabwean child health. In particular, political violence causes children to weigh 0.720 and 0.565 standard deviations less for their age and height, respectively, when they are exposed to it. Our research recommends adopting timely and effective initiatives to minimize the deleterious consequences of political violence.
\end{abstract}

